\title{
Energy gain induced by boundary crisis
}

\author{
C. Vieira Abud ${ }^{1,2, *}$ and R. Egydio de Carvalho ${ }^{2, \dagger}$ \\ ${ }^{1}$ Instituto de Física, Universidade de São Paulo-USP, 05315-970 São Paulo, São Paulo, Brazil \\ ${ }^{2}$ Instituto de Geociências e Ciências Exatas, Universidade Estadual Paulista-UNESP, 13506-900 Rio Claro, São Paulo, Brazil
}

(Received 27 May 2011; revised manuscript received 1 August 2011; published 8 September 2011)

\begin{abstract}
We consider a nonlinear system and show the unexpected and surprising result that, even for high dissipation, the mean energy of a particle can attain higher values than when there is no dissipation in the system. We reconsider the time-dependent annular billiard in the presence of inelastic collisions with the boundaries. For some magnitudes of dissipation, we observe the phenomenon of boundary crisis, which drives the particles to an asymptotic attractive fixed point located at a value of energy that is higher than the mean energy of the nondissipative case and so much higher than the mean energy just before the crisis. We should emphasize that the unexpected results presented here reveal the importance of a nonlinear dynamics analysis to explain the paradoxical strategy of introducing dissipation in the system in order to gain energy.
\end{abstract}

DOI: 10.1103/PhysRevE.84.036204

PACS number(s): 05.45.Ac, 05.45.Pq, 45.90.+t

\section{INTRODUCTION}

Almost all real systems in physics are essentially dissipative; they usually depend on mechanisms involving frictional forces or inelasticity and the meaning of weak or strong dissipation seems to be specific for each system. Recently, dissipative systems have received considerable attention in works that take advantage of the presence of the dissipation or of its elimination [1-3]. The role of dissipation has also been the object of analyses of broad interest in different branches of science [4-6]. In our case, where the dissipation is controlled by a parameter, we define weak dissipation as the regime in which an initial condition spends a long time in the same region, where there was an invariant curve in the nondissipative case, before being attracted by an attractor. On the other hand, if this transient is negligible so that the trajectory heads for rapidly to an attractor, we say the regime is one of strong dissipation. Even though these definitions are qualitative and system dependent, we introduce a dissipation coefficient $\gamma \in[0,1]$, in such a way that if $\gamma$ is close to 1 the dissipation is considered weak. In previous work [7], we have shown that we can increase the mean energy of a particle when a very weak dissipation, $\gamma \sim 0.99993$, is introduced in the system. The explanation for that mechanism is the creation of an attractor in the phase space whose ordinate is the particle velocity, at a higher velocity than the mean velocity of the nondissipative case. So, for all particles with low initial velocities, the attractor drives them to higher velocities. In the current paper, we use a smaller dissipation coefficient, $\gamma \sim 0.86$, which leads to a completely different topological phase space. Surely, there are many other regimes with stronger dissipation, but considering the effect already produced by $\gamma \sim 0.86$ we will call this case a regime of strong dissipation. In general, for dissipative systems, the emergence of a chaotic attractor as a parameter is varied is common. This chaotic attractor can suffer qualitative changes when it collides with an unstable invariant set. Such events are called crises, and they are created when a heteroclinic tangency occurs and moves to a transverse

\footnotetext{
*cabud@if.usp.br

${ }^{\dagger}$ Corresponding author: regydio@rc.unesp.br
}

heteroclinic intersection. As dissipative systems present some kind of attractor, the occurrence of crises is possible when, in addition to a chaotic attractor, at least one other attractor is present. Crises impose sudden changes in the chaotic attractor and they are typically classified in three types [8-15]: (i) fusion, when two or more chaotic attractors merge to form just one chaotic attractor; (ii) interior crises, when the chaotic attractor collides with an unstable periodic orbit, which is contained inside the basin of attraction of the same attractor; and finally (iii) boundary crises, when the boundary of the basin of attraction of the chaotic attractor coincides with the stable manifold of an external unstable invariant set. At this time the chaotic attractor is destroyed and it is replaced by a chaotic transient.

As an extension of our previous work [7], we are now going to investigate the effect of a boundary crisis on the mean energy of a particle in the limit of strong dissipation. We will show an abrupt and unexpected quantitative change in the mean velocity of a particle due to a boundary crisis.

The paper is divided as follows: In Sec. II we comment briefly about the geometry of the static annular billiard and we also introduce the time-dependent case. In Sec. III we introduce dissipation in the time-dependent billiard, while in Sec. IV we present our results and in Sec. V we conclude the paper.

\section{THE TIME-DEPENDENT NONDISSIPATIVE ANNULAR BILLIARD}

The static annular billiard consists of two circumscribed circles where the annular area is the accessible region for the motion of a free particle. The radius of the outer circle is assumed to be $R=1$, while the radius of the inner circle is called $r$. The distance between the centers of the two circles is the eccentricity and we call it $\delta$. In fact, the eccentricity plays the role of the perturbation parameter and it is responsible for the emergence of chaotic orbits. The constraint $(r+\delta)<$ 1 is always used, which implies that the inner circle cannot touch the external one. Inside the annulus, a particle moves freely along a straight line with constant kinetic energy until it collides elastically with the boundaries. After suffering a collision, the particle is specularly reflected, i.e., the incidence 


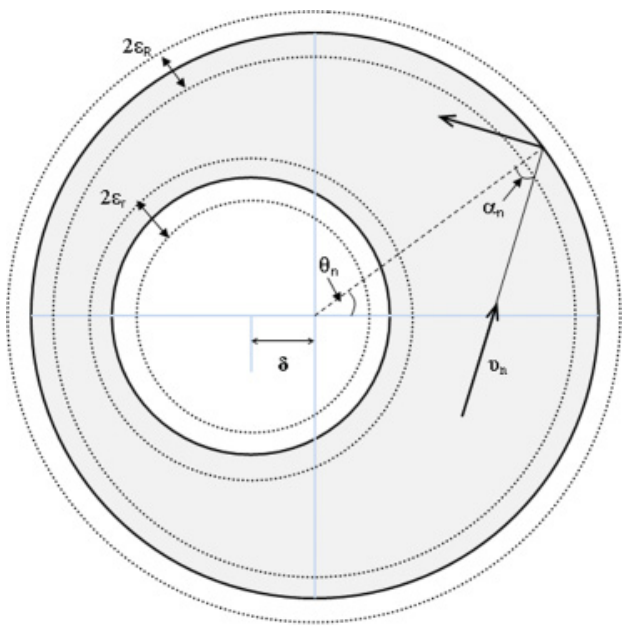

FIG. 1. (Color online) Generic illustration of the time-dependent annular billiard. The collision zones are the regions between the dotted circles. Throughout this work we keep $\delta=0$ and consider only the concentric case.

angle is equal to the reflection angle. The position of the particle is completely determined by the two angles $(\alpha, \theta)$. Here $\alpha$ is the incidence angle measured in relation to the normal of the outer circle and it is in the range $[-\pi / 2, \pi / 2]$. $\theta$ is associated with the arclength measured on the abscissa axis, inside the range $[-\pi, \pi]$. The bold circles of Fig. 1 illustrate the static billiard. The particle can develop two kinds of motion, which we split into two types: case $A$, when, between two successive collisions with the outer circle, the particle does not collide with the inner one; and case $B$, when after suffering a collision with the outer circle, the particle hits the inner circle before again reaching the outer circle. These movements are distinguished by using geometrical arguments; and the tangency condition $[16,17]$ occurs as

$$
\left|R \sin \left(\alpha_{n}\right)-\delta \sin \left(\theta_{n}-\alpha_{n}\right)\right|<r .
$$

This condition must be evaluated for all iterations of the static maps. So, in the static case, the dynamics is generated by nonlinear area preserving maps, whose canonical coordinates we define as $(L, S)$, with $L=\theta / 2 \pi$ and $S=\sin (\alpha)$, in such way that $-1 / 2 \leqslant L \leqslant 1 / 2$ and $-1 \leqslant S \leqslant 1$. When $\delta=0$, which corresponds to the concentric case, the system is globally integrable because in addition to the energy, the angular momentum of the particle is also conserved and the phase space of either case $r=0$ or $r \neq 0$ is filled by straight lines parallel to the $L$ axis, which correspond to periodic or quasiperiodic solutions. On the other hand, for the eccentric case $\delta \neq 0$, chaotic orbits and resonance structures, also known as Kolmogorov-Arnold-Moser (KAM) islands, emerge in the phase space.

In the time-dependent case, the billiard geometry can also be concentric or eccentric; moreover, the boundaries are not static any more. Now they oscillate in the radial direction around their position in the static case, defining a region called the collision zone. When the particle goes into the collision zone it can suffer one, many, or no collisions with the corresponding boundary (none, only for the inner circle). Due to the time dependence, the particle energy is not conserved any more and a second canonical phase plane is defined through the particle velocity and the phase oscillation of the outer boundary. We call it the energy phase plane. Hence, for the eccentric and time-dependent case, the phase space is in fact four dimensional (4D). However, if the circles are concentric, the particle angular momentum will be conserved and hence we can look only at the energy phase plane to understand the whole dynamics.

So breathing boundaries oscillate according to the equations

$$
R(t)=1+\varepsilon_{R} \cos (\omega t+\varphi), \quad r(t)=r+\varepsilon_{r} \cos (t+\phi),
$$

where $\omega$ is the ratio between the two frequencies, which we set to 1 . The quantities $\left(1, \varepsilon_{R}, \varphi\right)$ and $\left(r, \varepsilon_{r}, \phi\right)$ correspond to the static radii, amplitudes, and phases of the outer and the inner boundaries, respectively. The collisions between the particle and the boundaries can occur in the collision zones $\left[1+\varepsilon_{R}\right.$, $\left.1-\varepsilon_{R}\right]$ for the outer circle and $\left[r+\varepsilon_{r}, \mathrm{r}-\varepsilon_{r}\right]$ for the inner circle. After a collision, the particle can gain or lose energy and the dynamics is represented by a four-dimensional map given by sequences of impacts of the particle with the outer circle. The map corresponds to the application $\left(\theta_{n+1}, \alpha_{n+1},\left|v_{n+1}\right|, \varphi_{n+1}\right)=M\left(\theta_{n}, \alpha_{n},\left|v_{n}\right|, \varphi_{n}\right)$, where $\varphi$ is the phase of the external oscillating boundary, $v$ is the particle velocity, and the angles $\theta$ and $\alpha$ have been previously defined. In fact, the coordinates that we have used for the energy phase plane are $\left(\varphi,-v_{\eta}\right)$, where $v_{\eta}$ is the radial particle velocity. Nevertheless, we emphasize that in this current paper we consider only the concentric geometry, $\delta=0$, so that the particle angular momentum with respect to the center of the circles is a constant of motion and the nondissipative system is near-integrable. Thus it is computationally convenient to use the angular momentum $l$ as a system parameter. To iterate the map we have to give the initial condition $\left(\theta_{n}, \alpha_{n}, v_{n}, \varphi_{n}\right)$. However, as a consequence of the angular momentum preservation, the initial condition $\alpha_{n}$ can be obtained from the equation $|\vec{l}|=\left|\vec{r}_{0} \times \vec{v}_{0}\right|=\left|\vec{r}_{0}\right|\left|\vec{v}_{0}\right| \sin \left(\alpha_{n}\right)$, where $r_{0}$ comes from Eq. (2) with $t=0$ and $v_{0}$ is the total particle velocity. Consequently, $\sin \left(\alpha_{n}\right)=l /\left[1+\varepsilon_{R} \cos \left(\varphi_{n}\right)\right] v_{n}$ and we set the range for $l$ as $l \in\left[0, \varepsilon_{R}\right]$. Throughout this work we have arbitrarily chosen $l=0.01$ and $\theta_{n}=0.2$.

The whole map for the time-dependent annular billiard was developed in previous papers [18,19] and it is too long to be thoroughly reproduced in this paper. Therefore, readers who wish to know the algebraic calculations of the time-dependent annular billiard should consult $[18,19]$, and references cited therein, where all details are presented. To develop the numerical calculations of this current work, we compute all formulas of Secs. II and III of [19], and next we introduce dissipation through inelastic collisions of the particle with the boundaries as described in Sec. III below.

We emphasize that the tangency condition presented in Eq. (1) now should be replaced by

$$
\left|R(0) \sin \left(\alpha_{n}\right)-\delta \sin \left(\theta_{n}-\alpha_{n}\right)\right|<\left(r+\varepsilon_{r}\right),
$$

where $R(0)$ is the radius of the external boundary at the zero time given by the driving law in Eq. (2). In the numerical calculations we need the flight time, which is the time that a 
particle spends between two collisions with the outer circle. To calculate the flight time correctly, we restart the time at each collision with the external boundary, defining what we call the zero time. At that time, $R(0)=1+\varepsilon_{R} \cos (\varphi)$. We emphasize that we do not need to calculate the inner radius at a zero time, because the tangency condition is evaluated only if the particle goes into the collision zone. Hence, the right side of formula (3) should contain only the radius of the inner circle, plus the oscillation amplitude of the inner boundary. Thus, the orbits can still develop two kinds of motion, the cases A and B discussed above. However, now the particles may suffer successive collisions with the boundaries when they cross the collision zones. For the inner collision zone, a particle can also suffer no collision depending on its velocity and on the boundary position. In Fig. 2(a) we show a typical energy phase space for the case without dissipation, which will be useful to compare with the dissipative version.

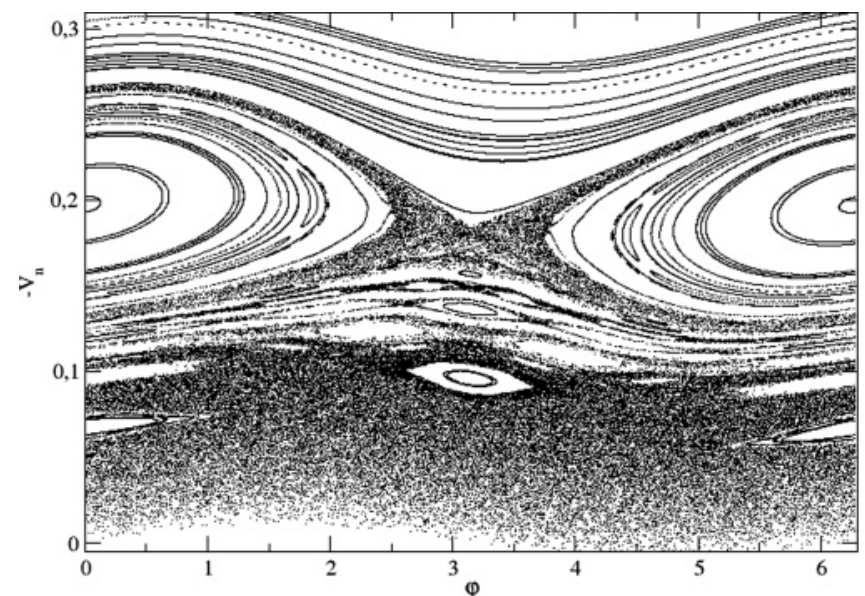

(a)

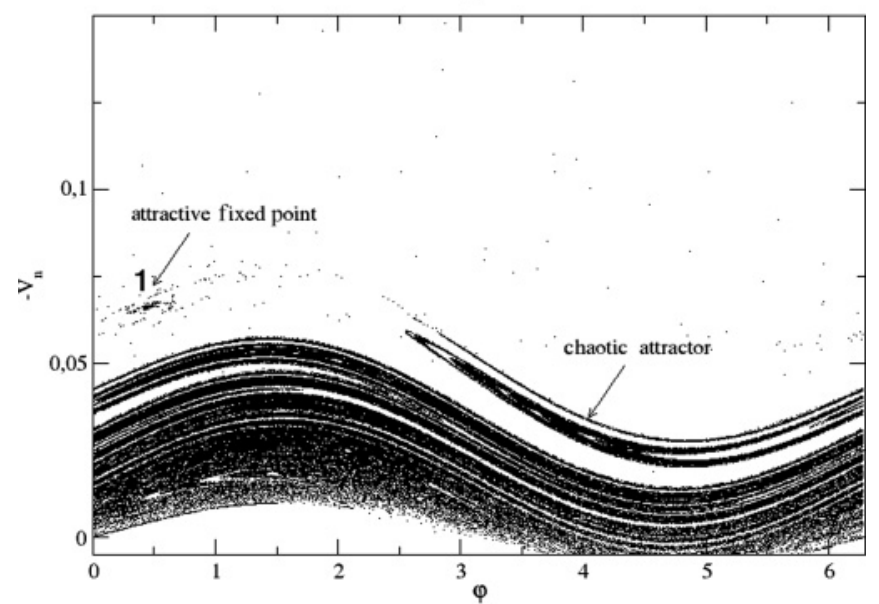

(b)

FIG. 2. Energy phase planes for the time-dependent annular billiard with $l=0.01$ and $\theta_{n}=0.2$. (a) The nondissipative, elastic, case $(\gamma=1)$. (b) The strongly dissipative case $(\gamma=0.8651)$. Almost all regular structures of the elastic case have been destroyed by the dissipation. In both cases (a) and (b) we have used $\varepsilon_{R}=\varepsilon_{r}=0.01$, $(\varphi-\phi)=0, r=0.4, \delta=0$ (concentric case), and the same initial conditions.

\section{INTRODUCING DISSIPATION}

In order to introduce dissipation in the time-dependent annular billiard, we now consider inelasticity in the collisions with the boundaries. In the collisions with both circles, the particles have their energies recalculated. We point out that no drag forces, or other kinds of dissipation, are present in the system.

In each collision, with any boundary, the momentum transfer due to the inelastic collisions is similar to that in the central force problem, i.e., in a collision the velocity components $\eta$ (radial) and $\tau$ (tangential) are given by

$$
V_{(n+1) \eta}=-\gamma V_{n \eta}+(1+\gamma) U, \quad V_{(n+1) \tau}=V_{n \tau},
$$

where $U$ corresponds to the velocity of the corresponding boundary where the collision occurred, which can be found from the time derivative of Eq. (2) evaluated at a time $t$, which corresponds to the flight time between two collisions with the outer circle. The parameter $\gamma \in[0,1]$ is the dissipation coefficient that acts only in the instant of the collision. The full inelastic movement (just one collision) happens when $\gamma=0$ and it will not be considered. In the opposite limit $\gamma=1$, the nondissipative case is recovered. Equations (4) show us that only the radial component is changed by the inelasticity.

In [7] we studied the dynamics in the limit of very weak dissipation and observed a bifurcation, in the energy phase plane, where a stable focus, for high velocity, was created from an elliptic fixed point. This focus is the main attractor and it drives the flux toward it. When there is no dissipation in the system, the elliptic fixed point is inside a resonance island, which is embedded in the chaotic sea and below a regular region with invariant tori. In addition to the cited bifurcation, the dissipation also destroys these invariant tori. So a particle starting with low velocity, in principle, could reach some regions of high velocities, which were blocked by the invariant tori. We considered an ensemble of low-velocity particles and evaluated the mean velocity of this ensemble after a very high iteration number. We got a nonintuitive result: for an interval of the parameter $\gamma$, the particle mean velocity for the dissipative case was asymptotically higher than in the nondissipative case. In addition, we proposed a general mechanism to gain energy from the introduction of dissipation: the creation of an attractor at high velocity scales from an island, embedded in the chaotic sea, whose energy is higher than the corresponding mean energy of the elastic system, for the same ensemble of initial conditions. However, this effect disappeared when we introduced a slightly stronger dissipation. The flux was driven to a low-velocity attractor. This study naturally led us to the current work. We would like to point out that our maps are 4D and we iterate all coordinates to define a trajectory. However, as we are interested in analyzing the behavior of the particle energy, the energy phase plane is sufficient for our purpose. The other canonical phase plane (called the geometric phase plane) would give us only the attractors' positions in the billiard plane. We could plot it, but no relevant information would emerge about the energy gain from a crisis. The bifurcation investigated in the current paper, in principle, can occur for any dimension. Even though the authors of [20] did not investigate this effect, it probably can occur since the model used there, the Fermi accelerator, is a particular case of the annular billiard, 
but with smaller dimension. In the next section we present and discuss our results.

\section{RESULTS AND DISCUSSION}

In our previous paper [7], we used a simplified model of the time-dependent annular billiard [18] to show that a very weak dissipation, combined with an appropriate parameter set, may induce an increase of the mean energy of the system when compared with the nondissipative case. In fact, we observed that the fixed points of the regular islands became attractors through bifurcations of elliptic fixed points. If one or some of these islands are embedded in a limited chaotic sea and located in regions whose energies are higher than the mean energy of the elastic system, then the mechanism for gaining energy is established. In the cited simplified model the billiard boundaries are kept static, but in the instant of a collision the boundaries transfer momentum to the particle as if they were oscillating. This is a mathematical artifice, which leads to an easier computational calculation and presents good results. Thus, successive collisions are not expected in the simplified model. Even though this simplification does not change the mean properties of the system, and the results are reliable for weak dissipation and medium- to high-energy regimes, for strong dissipation the successive collisions become so relevant in launching back the particle, that we must consider the complete (or real) model [19]. We point out that we got the manifolds of the saddle points with very good resolution, in the real model, unlike our experience using the simplified model. Hence, the results we are going to present here consider only the real model. We also emphasize that, even though during the flight the particle can collide with the inner circle, we stored only the collisions with the outer circle.

The energy phase space is showed in Fig. 2 with the parameter set $\varepsilon_{R}=\varepsilon_{r}=0.01,(\varphi-\phi)=0, l=0.01$, and $\theta_{n}=$ 0.2 with $r=0.4$ and $\delta=0$. Figure 2(a) represents the elastic case, $\gamma=1$, and in Fig. 2(b) there is a strong dissipation, $\gamma=$ 0.8651 , both with breathing boundaries. Figure 2(a) shows a chaotic sea for low velocities with KAM islands and invariant tori that limit the energy growth of the particles starting in the chaotic sea. In the time-dependent annular billiard, these invariant tori could be broken by increasing the eccentricity of the circles and the mechanism of Fermi acceleration could happen $[18,19,21]$. However, throughout this work we dealt only with the concentric annular billiard. In Fig. 2(b) we show the phase space for the dissipative case with the same initial conditions used in Fig. 2(a), and we can observe that these initial conditions have been captured, either by the attractive fixed point or by the chaotic attractor. Due to the strong dissipation, the orbits quickly converge toward one of the attractors. In Fig. 2(b) we also plot the transient.

The existence of two different attractors (one attractive fixed point and one strange attractor) in the phase space allowed us to question if it was possible to observe crises in this system and what would be their role in the energy of the particles. A signature of a boundary crisis (B crisis) is the sudden destruction of the chaotic attractor. This event is common in physics [22-25] and it happens in dissipative systems because of the intersection of the stable manifold of a saddle point with the associated unstable manifold.

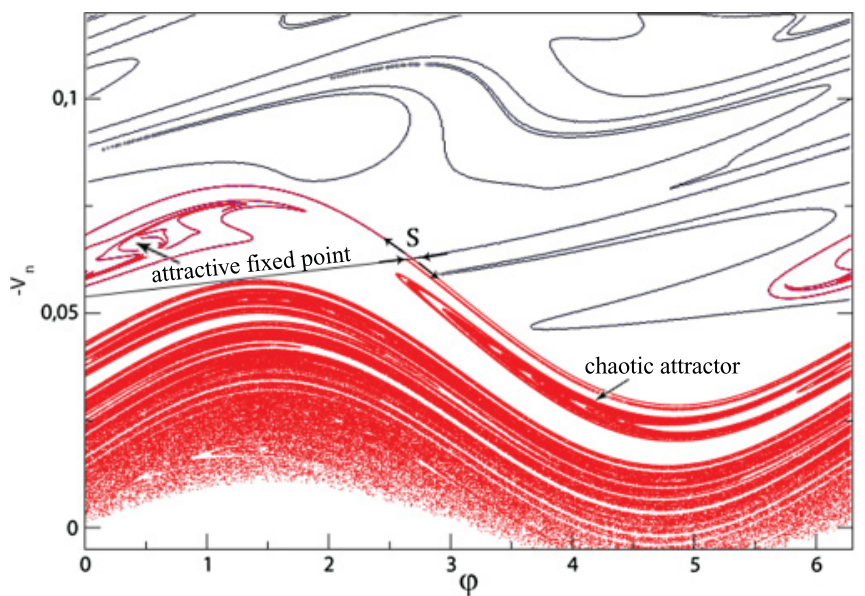

(a)

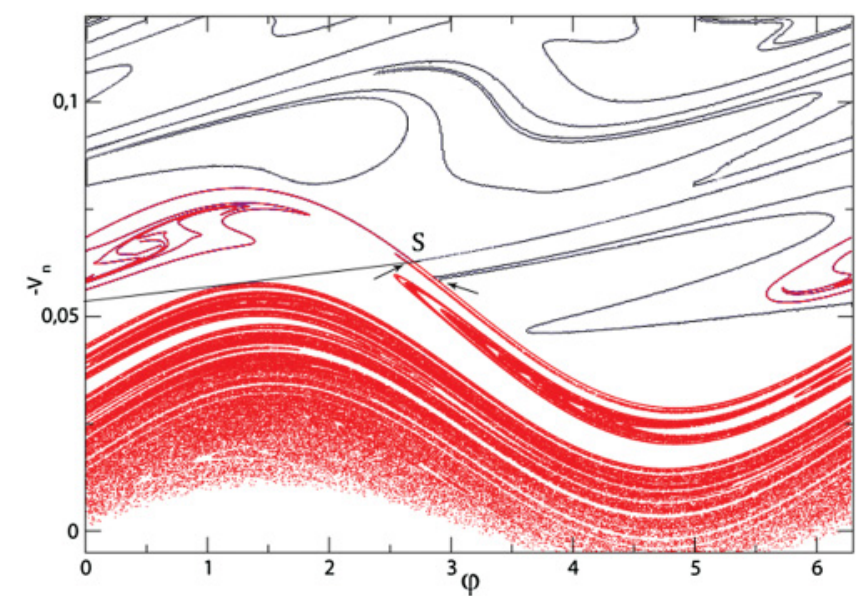

(b)

FIG. 3. (Color online) The stable and unstable manifolds of the periodic hyperbolic point $S$. The attractive fixed point is around: $-\mathrm{V}_{\eta} \approx 0.065$ and $\varphi \approx 0.50$. We have chosen $l=0.01$ and $\theta_{n}=0.2$ in all plots. (a) Before the B crisis, with $\gamma=0.8651$. (b) After the $\mathrm{B}$ crisis, with $\gamma=0.8671$. The arrows indicate the crossings of the stable and unstable manifolds.

We show in Fig. 3(a) the behavior of the branches of the manifolds (two stable and two unstable) of the saddle point identified by $S$ when we used the parameter set $\gamma=0.8651$, $\varepsilon_{R}=\varepsilon_{r}=0.01,(\varphi-\phi)=0, r=0.4$, and $\delta=0$ (concentric case). The stable manifolds were found from a contour plot of the basin of attraction. Due to the preservation of the angular momentum, even in the time-dependent case, the dynamics is restricted to a two-dimensional manifold of the 4D phase space.

Looking at Fig. 3(a), we observe that the saddle point $S$ has coordinates $-v \sim 0.065$ and $\varphi \sim 3$; thus the reader could ask about the origin of this saddle point since there is no similarity with the nondissipative case in Fig. 2(a). We emphasize that the dissipation destroys almost all invariant structures and turns the elliptical points into attractive foci. When the dissipation is weak there are many stable foci. However, as the dissipation magnitude increases, they are gradually destroyed. For the parameter set we are using, the last surviving attractive fixed point is the stable focus whose coordinates in the conservative energy phase space are $-v \sim 0.065$ and $\varphi \sim 0$. Thus, for 
the time-dependent annular billiard, the value $\gamma=0.8651$ corresponds approximately to the threshold for the existence of the last attractive fixed point. This means that the dissipative phase spaces studied in this work have only two attractors, one attractive fixed point and one strange attractor.

In that context, we observed numerically that the saddle point $S$ in Fig. 2 originated from a periodic hyperbolic point, which emerged in the chaotic sea in the nondissipative case. The numerical procedure to determine their unstable manifolds required a hard computational effort, because we have to find a tiny set of initial conditions around the $S$ point in order to follow the unstable manifolds. The method known as the sprinkler method is very convenient, because we do not need to start from the exact location of the hyperbolic point, and only a few iterations (fewer than 20) of each initial condition (we used a grid of $500 \times 500$ ), are necessary to draw the unstable manifolds. We succeeded with this method, starting with the coordinates $(v, \varphi)=(0.06593,2.65602)$ as the center of the square grid around the point $S$. This means that this pair of coordinates corresponds to a very good approximation for the coordinates of the $S$ point in Fig. 3 .

From Fig. 3 we can see that the upper unstable manifold follows toward the attractive fixed point while the bottom one defines the chaotic attractor. On the other hand, the two stable manifolds generate the boundaries of the attractive fixed point, splitting the initial conditions, which follow toward the stable focus, from those heading for the chaotic attractor. A slight increasing of the dissipation intensity, $\gamma=0.8671$, is implied in the collision of the border of the chaotic attractor (stable manifold) with the unstable manifold. That is represented by arrows in Fig. 3(b), indicating the intersections. From this time, the basin of attraction of the chaotic attractor and the chaotic attractor do not exist any longer, because a B crisis has occurred. In the place of the chaotic attractor there is a chaotic transient, which can be understood as the interval of time before the initial conditions, departing from the region where there was the chaotic attractor, are captured by the attractive fixed point. Now, let us discuss why this event of crisis, despite being physically well known, can be intriguing at the same time. Before the B crisis, the particle velocities inside the chaotic attractor are limited by the velocities of the basin of attraction of the chaotic attractor. However, after the B crisis these velocities can grow up to the velocity of the attractive fixed point, which has higher energy.

We study the statistics of the mean velocities of an ensemble of particles. We take an ensemble of 500 low-velocity particles, for instance $v=0.02$, and randomly sort the phase $\varphi$, keeping $l=0.01$ and $\theta_{n}=0.2$ in all plots. Next, we evolve one iteration of all initial conditions and evaluate the average velocity for one iteration. We repeat this average process for two iterations of all initial conditions and so on for all iterations, up to $5 \times 10^{6}$. In Fig. 4 we show the behavior of the average velocities for the following three cases: the conservative case $\gamma=1$, before the crisis $\gamma=0.8651$, and after the crisis $\gamma=$ 0.8671 . The chaotic attractor is destroyed immediately after the $\mathrm{B}$ crisis. The difference in the dissipation intensity before and after the $\mathrm{B}$ crisis is around $0.2 \%$, but this small difference has produced a surprising $\sim 160 \%$ of energy gain, because before the Bcrisis the mean velocity was 0.0251 and after the Bcrisis it is 0.0655 , as shown in Fig. 4. Moreover, the

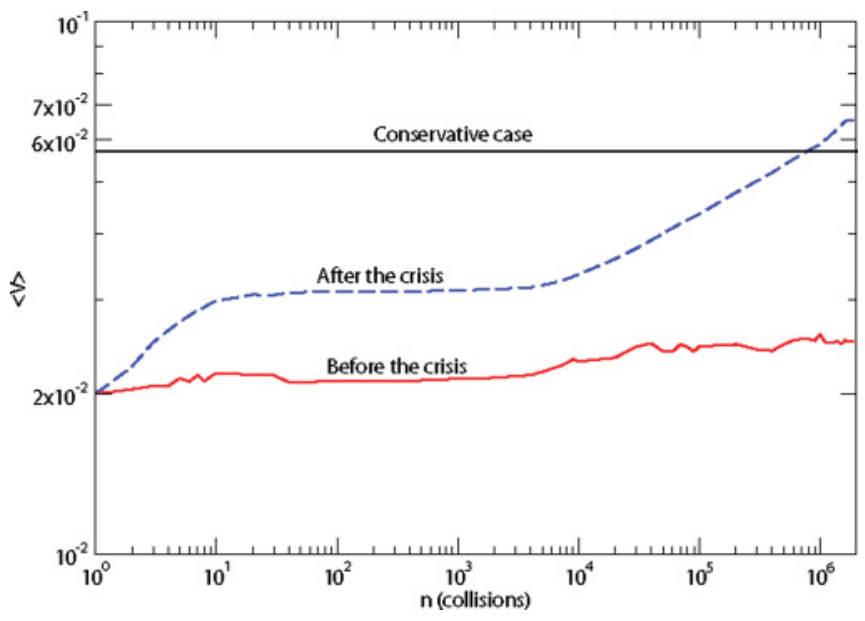

FIG. 4. (Color online) The average velocities for three events: for the conservative case, with $\gamma=1$; before the B crisis, with $\gamma=0.8651$; after the B crisis, with $\gamma=0.8671$. The values of the mean energies after $5 \times 10^{6}$ iterations are $5.69 \times 10^{-2}$ in the conservative case, $2.51 \times 10^{-2}$ before the $\mathrm{B}$ crisis, and $6.55 \times 10^{-2}$ after the $\mathrm{B}$ crisis.

new energy after the B crisis also is higher than the energy of the conservative case, even considering strong dissipation. The energy gain in this case is around $15 \%$. In our previous paper [7], the creation of an attractive fixed point at high velocity on the phase space was responsible for driving the system to a regime of higher energy. This occurred with a very weak dissipation. In the current paper, the mechanism of energy gain is the destruction of a chaotic attractor by a crisis event, and this occurs in a regime of very strong dissipation. In addition to the difference in the mechanisms, the nonlinear dynamics structures of the corresponding phase spaces are really different. Hence, in this case of strong dissipation, the mechanism which allows energy gain is composed of two ingredients, a B crisis and a high-velocity attractive fixed point. It is important to point out two considerations of the conservative case. First, note that the procedure to calculate the average, as mentioned above, takes into account only particles starting with low velocities, which means velocities only from the chaotic component [see Fig. 2(a)]. Second, the evaluation of the mean energy of the nondissipative case spends too much time around the islands due to the successive stickiness around them [26-29]. Even though the ergodicity of the chaotic sea of the nondissipative case is not algebraically demonstrated, we have observed numerically that when we iterate the system for a very long time, on the average, the trajectories do not spend more time close to these islands than to other regions of similar volumes. When we say "average energy of the system," in fact we are referring to the average energy reached by those particles that started with low energy inside the chaotic sea or the chaotic attractor. In the nondissipative case, the mean energy is approximately one-half of the height of the chaotic sea. For the dissipative case, after the B crisis, the energies saturate at the value of the attractive fixed point energy. We study only the chaotic region with low energy because we are interested to observe the conditions in which a particle can gain energy.

Another interesting and important point which we will address here concerns the possibility of having families of 


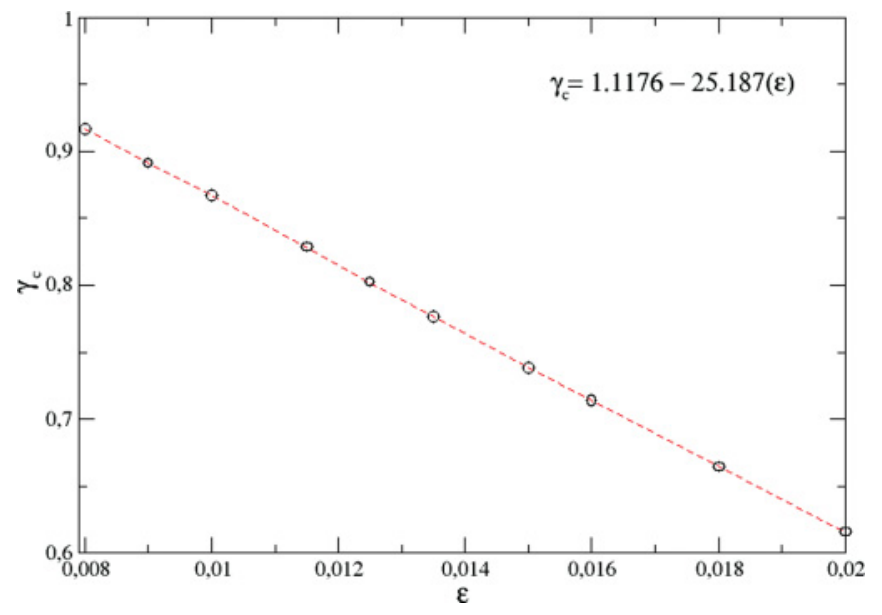

FIG. 5. (Color online) The critical dissipation coefficients, for crises, versus the amplitude of oscillation coefficients. Each point $\left(\varepsilon_{R}, \gamma_{c}\right)$ below the dashed line indicates that a crisis event will happen.

boundary crises. As a B crisis has occurred for a particular set of parameters, then we supposed it would be possible for the system also to present crises for other parameter sets. In order to investigate this point, we kept the same values for both boundary amplitudes of oscillation, $\varepsilon_{R}=\varepsilon_{r}$, and we considered $\varepsilon_{R}$ as a changeable parameter. As we varied $\varepsilon_{R}$, we searched for the critical dissipation coefficient $\gamma_{c}$ at which a crisis could occur. We point out that for each value of $\varepsilon_{R}$ we have a different dynamics in the energy phase space. However, the region labeled 1 in Fig. 2(b), where the attractive fixed point was created, is nearly located at the same place for the parameter set we have used. So we searched for the dissipation coefficient, for initial conditions starting inside the chaotic attractor, which allowed them to reach the attractive fixed point. Figure 5 shows the critical dissipation coefficient as a function of $\varepsilon_{R}$. We varied this amplitude in the range $[0.008,0.02]$, and we got the numerical linear law $\gamma_{c}=1.1176-25.177 \varepsilon_{R}$ with very good precision. With this expression and the range of values for the amplitude, the dissipation coefficient falls approximately in the range $[0.61,0.92]$. The phase space is essentially limited by a lowvelocity chaotic attractor and an attractive fixed point with larger velocity. So we obtained a two-parameter family of crises, all of them leading to energy gain no matter how strong the dissipations are.

\section{CONCLUSION}

We have shown that a particle can gain energy in the context of very strong dissipation and also that an event of crisis is responsible for this energy gain. The explanation for this apparent paradox comes from nonlinear dynamics tools. We stated that a strongly dissipative system of noninteracting particles can experience the mechanism of energy gain if (i) the chaotic attractor (from where the particles will start) is confined in the energy phase plane, (ii) in this phase plane there is at least one stable focus with velocity higher than the average velocity of the chaotic component, and (iii) the dissipation is controlled by a parameter which can induce a B crisis in the system. In fact, the other kinds of crisis, fusion or interior, also could lead to energy gain, since they generate an expansion of the original attractor.

It is worth commenting that in the pulsating elastic case, $\gamma=1$, with concentric circles $(\delta=0)$, the chaotic sea exists in the energy phase space for low energies and it is limited by invariant tori. Hence, the mean energy of the chaotic region can be evaluated simply by initializing an ensemble of initial conditions inside the chaos, which will be approximately one-half of the chaotic column. So the energy gain in this case will be associated with the size of the chaotic sea. This can be achieved by changing some parameter, such as the radius of the inner circle $(r)$ or the amplitudes of the oscillations $\left(\varepsilon_{r}\right.$ or $\left.\varepsilon_{R}\right)$, which will naturally enlarge the chaotic sea. On the other hand, for $\gamma=1$ but in the eccentric case, the system can experience Fermi acceleration as already reported in Refs. $[18,19]$. We emphasize that, if the ensemble of initial conditions started with velocities higher than the mean velocity of the chaotic component, obviously the particles would not gain energy. Even in the eccentric case when $\delta \neq 0$, any magnitude of dissipation would break down the mechanism of Fermi acceleration, in agreement with the results presented in [30].

Even though we have obtained our results by considering inelastic collisions, it is still not clear to us if other kinds of dissipation (such as friction or viscosity) would produce the same effect. We believe that if the corresponding dynamics of the nondissipative case presents resonance structures and chaos, and in the dissipative version at least a stable focus and a chaotic attractor occur, then this mechanism could also be observed.

Although our approach has been developed in a particular model, our results constitute a powerful tool for many scientific problems and they emphasize how important has been the development of nonlinear dynamics in order to unveil some classical paradigms. So we consider that our results after spreading broadly will contribute positively to other fields of science.

\section{ACKNOWLEDGMENTS}

The authors gratefully acknowledge fruitful discussions with Professor Iberê L. Caldas and Professor Elbert E. N. Macau and thank the Brazilian scientific agencies FAPESP, through the Processes No. 06/51653-0 and No. 07/57992-4, and CAPES for financial support.
[1] D. Pfenniger and C. Norman, Astrophys. J. 363, 391 (1990).

[2] T. Cubaud and T. G. Mason, Phys. Rev. Lett. 96, 114501 (2006).

[3] K. Yu Bliokh et al., Phys. Rev. Lett. 97, 243904 (2006).

[4] C. F. Gmachl, Nature (London) 467, 37 (2010).
[5] L. Mayer, S. Kazantzidis, A. Escala, and S. Callegari, Nature (London) 466, 1082 (2010).

[6] T. Rudel, O. Kepp, and V. Kozjak-Pavlovic, Nature Rev. Microbiol. 8, 693 (2010). 
[7] R. Egydio de Carvalho, C. V. Abud, and F. C. Souza, Phys. Rev. E 77, 036204 (2008).

[8] C. Grebogi, E. Ott, and J. A. Yorke, Physica D 7, 181 (1983).

[9] C. Grebogi, E. Ott, and J. A. Yorke, Phys. Rev. Lett. 48, 1507 (1982).

[10] C. Grebogi, E. Ott, F. Romeiras, and J. A. Yorke, Phys. Rev. A 36, 5365 (1987).

[11] H. B. Stewart, Y. Ueda, C. Grebogi, and J. A. Yorke, Phys. Rev. Lett. 75, 2478 (1995).

[12] J. A. Gallas, C. Grebogi, and J. A. Yorke, Phys. Rev. Lett. 71, 1359 (1993).

[13] C. Simó, J. Stat. Phys. 21, 465 (1979).

[14] M. Benedicks and L. Carleson, Ann. Math. 133, 73 (1991).

[15] E. Ott, Chaos in Dynamical Systems (Cambridge University Press, Cambridge, 1993)

[16] N. Saitô, H. Hirooka, J. Ford, F. Vivaldi, and G. H. Walker, Physica D 5, 273 (1982).

[17] O. Bohigas, D. Boosé, R. Egydio de Carvalho, and V. Marvulle, Nucl. Phys. A 560, 197 (1993).
[18] R. Egydio de Carvalho, F. C. Souza, and E. D. Leonel, J. Phys. A 39, 3561 (2006).

[19] R. Egydio de Carvalho, F. C. Souza, and E. D. Leonel, Phys. Rev. E 73, 066229 (2006).

[20] E. D. Leonel and R. Egydio de Carvalho, Phys. Lett. A 364, 475 (2007).

[21] E. Fermi, Phys. Rev. 75, 1169 (1949).

[22] C. Jeffries and J. Perez, Phys. Rev. A 27, 601 (1983).

[23] D. Dangoisse, P. Glorieux, and D. Hennequin, Phys. Rev. Lett. 57, 2657 (1986).

[24] C. Abraham et al., Chaos, Solitons Fractals 24, 869 (2005).

[25] H. Kantz and P. Grassberger, Physica D 75, 17 (1985).

[26] G. M. Zaslavsky, Phys. Rep. 371, 461 (2002).

[27] G. M. Zaslavsky, Hamiltonian Chaos and Fractional Dynamics (Oxford University Press, Oxford, 2005).

[28] G. Contopoulos and M. Harsoula, Int. J. Bifurcation Chaos Appl. Sci. Eng. 18, 2929 (2005).

[29] E. G. Altmann, A. E. Motter, and H. Kantz, Phys. Rev. E 73, 026207 (2006).

[30] E. D. Leonel and L. A. Bunimovich, Phys. Rev. Lett. 104, 224101 (2010). 